\title{
Research on Customer Relationship Management System Based on Cloud Computing Platform
}

\author{
Liu Yang \\ Chongqing College of Electronic Engineering,Chongqing,China \\ 173676096@qq.com
}

Keywords: Customer Relationship Management System, Cloud Computing Platform

\begin{abstract}
Customer relationship management system can help companies manage the relationship between businesses and customers. With customer relationship management system, companies can interact with the unified management of sales, marketing and other departments and customers, to explore more business opportunities. With the burgeoning cloud computing, customer relationship management systems are also being developed toward the direction of cloud computing. Cloud-based customer relationship management system for SMEs brought hope, SMEs will be able to enjoy the customer relationship management system brings convenience, thereby enhancing the competitiveness of enterprises. But the application of the new system has also brought new security risks, this is an important issue cloud customer relationship management system based on the urgent need to resolve.
\end{abstract}

\section{Introduction}

With the rapid development of information technology in recent years, companies are subject to all aspects of a huge impact. Companies need customer needs, changes in the market to respond positively. The traditional business model began to shift to a customer-centric business model. Customers as the most valuable resources entered into the enterprise management system. Resources companies should focus on customer-centric arrangements. Who promptly determine market trends find out the needs of customers, who can occupy a place in the fierce competition in the market. In order to better communicate with customers, providing customers with convenient purchase channels, provide better customer service, provide better customer care, and produce customer relationship management. One of its aims is to is to help businesses more effectively manage the sales process cycle: with concurrency and potential customers, maintain existing customers, providing customers with better service and enhance the relationship between businesses and customers. And the rational use of marketing tools category, providing innovative and personalized customer communications and services, related to the use of information technology and data systems, such as data mining and marketing to coordinate sales, marketing and support services to the enterprise and its customers' interaction. Therefore, customer relationship management system is increasingly becoming an important part of enterprise information.

Cloud computing is in the previous grid computing, distributed computing, a new mode of development, based on the polymerization it a lot of computing resources, storage resources together to form a huge pool of shared resources, can provide users with demand taken resources and flexible scalability. Cloud computing has the convenience, economy, high scalability and other advantages, thus causing more and more business concerns. With cloud computing, companies can get rid of IT facilities management and maintenance of heavy pressure, which will focus on their own core business. In the information industry, it is generally believed Cloud computing is another important growth area following the information industry since the wave of the Internet, has great market potential. According to International Data Corporation IDC predicts the next few years in business spending on cloud computing will surge to 2013 is expected to reach $\$ 42$ billion in size, accounting for the total amount of IT spending growth in a quarter. In addition, because the development of the concept of cloud computing with the current trends respected green economy consistent, it has also been a strong advocate and promotes world countries and regions. Cloud 
computing represents the current information industry towards centralized, large-scale, professional development trend, so some people likened to cloud computing today industrial revolution taking place in the information industry.

From the foregoing, enterprise users to deploy their own system application to the cloud computing platform, not only reduces the cost of enterprise IT can also be flexible to get more resources from cloud computing platforms in the enterprise business growth in order to meet the business growth needs.

\section{The Concept of Cloud computing}

Cloud computing as an economy of scale to promote the network computing model, which integrates a large number of computing, storage, software and other resources to form a huge pool of virtual resources as a basis for the user to provide economic, convenient and flexible service. It combines the use of virtualization, distributed technology, it is a new service different from the traditional way. Cloud computing will extend from the desktop computing power of the Internet, it is a kind of network computing unlimited scalability.

It has the following five main features: on-demand service taken: Users can according to their needs, request the resources required, such as computing resources, network bandwidth resources; many types of network access: support a variety of client access access, such as smart phones, laptops, and servers; resources are concentrated: a lot of physical resources together, and available to users through a multi-tenant mode, the user can dynamically use physical and virtual resources, such as memory, hard disk storage. Flexibility: resources can be flexible and rapid application and release, you can even automatically be changed as needed. From a user perspective, almost unlimited resources, ready to meet their own needs. Measurable service: Cloud computing platform based on application load, automatic control and optimize the use of resources. You can monitor the use of resources, and provides reports for providers and users with a certain degree of transparency. For enterprises, business users can take advantage of the various resources in the cloud quickly build their own applications, add new features, remove unwanted features, easy stretching their application, to better meet the needs of enterprise customers. For individuals, the cloud may contain a wide variety of applications, it offers a wide variety of applications and services for individual users. Whether the user is using a personal computer or smart terminal can be connected to the cloud, it is convenient to complete the operation, make full use of computing power and storage capacity of the cloud.

\section{The Customer Relationship Management System}

Customer relationship management is to follow the customer-oriented concept, the customer is the systematic study, by improving customer service levels, and timely communication with customers, improve customer-related business processes, improve customer loyalty, retention old customers at the same time, the development of potential customers, and constantly strive for business opportunities, and ultimately the company continues to develop and maintain their competitive advantage in the fierce competition in the market. Customer relationship management system is to better customer relationship management and application management system. Customer relationship management system, which is mainly to solve the main problem the following aspects: Problems operational management, sales automation, customer service; customer service issues management, call center construction, channel construction; data management analysis, there is the construction of customer information databases, data mining, building the knowledge base and the like. By addressing these aspects, the customer as the core enterprise business processes through the combination of enterprise information management system, the accumulation of effective customer information, and use of customer knowledge acquired to meet customer needs and strive to achieve optimize existing resources configuration. Customer relationship management system from the business front, it provides collection, data analysis of customer information, enabling enterprises to leverage their existing customer resources, and expand into new markets and develop new business 
channels on the basis of the original enhance customer satisfaction and corporate profitability; from a business background, the background of the integration of enterprise resources, traditional enterprise management solutions and customer relationship management systems combine to achieve automation of internal business processes, management along the business processes of enterprises, improve productivity.

\section{The Security Risk of Customer Relationship Management System}

The traditional security issues facing customer relationship management system in this system is also present. Customer relationship management system as a customer-centric business automation platform to support communication and collaboration enterprise management personnel, sales personnel, customers, etc., a major role is between businesses and customers and internal information flow transfer carried by hand into automation, it is a multi-role management system. Where each user has a different system permissions, you can view or modify data in their own right within the system. (1) unauthorized access: unauthorized access also refers to the illegal access, generally refers to the user access to it should it not have access to data, resulting in data leakage. Unauthorized access generally have the following two situations: First, without a user authentication system (such as stolen valid user name and password illegal users) invade the system, access to data systems, resulting in data leakage; one is the system legitimate users after login to the system to access beyond their own access to sensitive data, resulting in leakage of sensitive data ultra vires. (2) denied the use of Service: After service is denied the use of legitimate users of the system to operate in the system, for some bad reasons, to deny their own operations are performed, which may cause some damage to the enterprise. (3) net a little eavesdropping, tampering: slightly network eavesdropping, tampering means that the attacker can transfer user information interact with the system, the use of technical means to obtain information transmitted and can be sent to the recipient prior to modification in the information to achieve malicious purposes. (4) Interrupt Attack: Interrupt attack is a malicious attacker intercepts the transmission of information and blocking its transmission, resulting information can not be transferred to the recipient. (5) denial of service: denial of service means that the system provides the user specifies an operation and complete, for some bad reasons, to deny their own operations are provided with a user's denied the use of a similar service.

\section{The Security of Customer Relationship Management System}

Security issues facing customer relationship management system are: unauthorized access, denied the use of services, network eavesdropping, tampering, interrupted the attack, denied service. For use denial of service, network tampering, denial of service security risk, can be resolved through digital signatures. Digital signature is similar to the real world of handwritten signatures, has good security, you can confirm the integrity of the information, with the non-repudiation. As long as the receiving party through a digital signature verification, we can confirm that the received information is true expression of the will of the sender. For the network eavesdropping, the transmission can be encrypted to prevent eavesdropping third party information transmitted. Interrupt attack risk can preclude the use of reliable handshaking protocols and mechanisms to ensure that the communication process is not interrupted. For unauthorized access, use access control mechanisms to resolve, but the current access control models have advantages and disadvantages.

For the risks faced by the CRM system itself, in order to prevent illegal users two kinds of systems, in addition to the basic user name and password login, should also authenticate the user's identity to prove the currently logged in user is a legitimate user, not illegal to steal user account. User to enter a user name and password when you log in, the system will enter the user name and password of a user with a user name and password stored in the database for comparison, verified user login information submitted is correct. For increased security, the user password is stored in the database is not explicit, but after a one-way hash function processes the hash value. In contrast 
to the system user name and password, no direct comparison plaintext password, the password is submitted by the user with the same hash function to give a hash value, and then with the hash value stored in the system compared to verify the user's password correctness. Using role-based access control for different users to manage, assign a role for each user, it can only limited access to the system to allow access to the data, which can prevent low-level user access to data based on dense high. In order to ensure speed and security encryption and decryption using symmetric encryption methods for data encryption and decryption using asymmetric encryption method used symmetric encryption key for encryption and decryption.

For security issues cloud computing platform, take virtual machine isolation. Cloud computing platform virtual machines through virtualization of hardware resources from the platform in each virtual machine can access to the underlying hardware resources. If there are loopholes lead to the virtual controller for each virtual machine can not be completely isolated, a virtual machine can access another virtual machine's storage and memory, it will cause great security problem. To address this security risk, use of strong access control technologies, virtual machine isolation, to prevent access to a virtual machine to another virtual machine storage and memory.

First, the security classification of virtual machines and virtual resources into three levels: confidential, secret and no security classification. Dense three security order: secret more than a secret, a secret no more than state secrets. Reached a certain level of security of virtual machines can dictate the same security classification or lower level virtual resources. Write control strategy as follows:

Read control: the security level of the virtual machine must not be less than the security level of the virtual resource being accessed

Control: the level of security of the virtual machine must not be higher than the security level is written to virtual resources. This prevents a high level of security resources to spread low-level security virtual machine, or spread the flow of resources can only higher level virtual machine security level consolidated virtual machine.

\section{Conclusions}

Cloud computing is an economy of scale to promote the network computing model, which integrates a large number of computing, storage, software and other resources to form a huge virtual resource pool, the users can provide convenient, economical, dynamic and scalable service. The core idea is that the resources and capabilities on the network more effectively share, to achieve high efficiency, low cost computing goals. The emergence of cloud computing provides the possibility to meet the various needs of CRM.

\section{References}

[1] Feng Dengguo: Journal of Software, Vol. 6 (2004) No 53, p.25-26

[2] Guo Qingjun: Modern Computer, Vol. 12 (2005) No 27, p.74-76

[3] Luo Hong: Computer and Network, Vol. 1 (2006) No 33, p.11-14

[4] Feng Dengguo: Network Security Technology and Application, Vol. 3 (2007) No33, p.121-124

[5] Zhang Chun: Journal of Changsha University, Vol. 6 (2004) No 53, p.25-26 